\title{
NIAID Mycoses Study Group Multicenter Trial of Oral Itraconazole Therapy for Invasive Aspergillosis
}

David W. Denning, MBBS, San Jose, California, Jeanette Y. Lee, PhD, Birmingham, Alabama, John S. Hostetler, MD, San Jose California, Peter Pappas, MD, Birmingham, Alabama, Carol A. Kauffman, MD, Ann Arbor, Michigan, Daniel H. Dewsnup, DO, San Jose, California, John N. Galgiani, MD, Tucson, Arizona, John R. Graybill, MD, San Antonio, Texas, Alan M. Sugar, MD, Boston, Massachusetts, Antonino Catanzaro, MD, San Jose, California, Harry Gallis, MD, John R. Perfect, MD, Durham, North Carolina, Bonita Dockery, RN, William E. Dismukes, MD, Birmingham, Alabama, David A. Stevens, MD, San Jose, California

BACKGROUND: Invasive aspergillosis is the most common invasive mould infection and a major cause of mortality in immunocompromised patients. Response to amphotericin B, the only antifungal agent licensed in the United States for the treatment of aspergillosis, is suboptimal.

METHODS: A multicenter open study with strict entry criteria for invasive aspergillosis evaluated oral itraconazole $1600 \mathrm{mg} / \mathrm{d}$ for 4 days followed by $400 \mathrm{mg} / \mathrm{d}$ ) in patients with various underlying conditions. Response was based on clinical and radiologic criteria plus microbiology, histopathology, and autopsy data. Responses were categorized as complete, partial, or stable.

Failure was categorized as an itraconazole failure or overall failure.

RESULTS: Our study population consisted of 76 evaluable patients. Therapy duration varied from 0.3 to 97 weeks (median 46). At the end of treatment, $30(39 \%)$ patients had a complete or partial response, and $3(4 \%)$ had a stable

From the Division of Infectious Diseases, Department of Medicine, Santa Clara Valley Medical Center, San Jose; and California Institute of Medical Research, San Jose; and Division of Infectious Diseases and Geographic Medicine, Stanford University School of Medicine, Stanford, California (DWD, JSH, DHD, DAS); and the Division of Biostatistics, Comprehensive Cancer Center (JYL); and Division of infectious Diseases, Department of Medicine (PP, BD, WED), The University of Alabama at Birmingham, Birmingham, Alabama; and the Division of Infectious Discascs, Department of Internal Medicine (CAK), University of Michigan Medical School, Veterans Administration Medical Center, Ann Arbor, Michigan; and the Division of Infectious Diseases, Medical Service (JNG), Veterans Affairs Medical Center and Department of Medicine, University of Arizona, Tucson, Arizona (JNG); and Division of Infectious Diseases, Audie Murphy Memorial Veterans Hospital and Department of Medicine, University of Texas Health Science Center, San Antonio, Texas (JRG); and the Division of Infectious Diseases, Department of Medicine, Boston University School of Medicine (AMS),

Boston, Massachusetts; and the Division of Pulmonary Medicine,

Department of Medicine (AC), University of California, San Diego, California; and the Division of Infectious Diseases, Department of Medicine (HG, JRP), Duke University, Durham, North Carolina.

Requests for reprints should be addressed to David A. Stevens, MD, the Division of Infectious Diseases, Department of Medicine, Santa Clara Valley Medical Center, 751 South Bascom Avenue, San Jose, California 95128.

This work was supported in part by grants to the Mycoses Study Group (N01-A1-15082) from the National Institutes of Health, National Institute of Allergy and Infectious Diseases, and Janssen Research Foundation.

Manuscript submitted August 9, 1993, and accepted in revised form December 23, 1993. response, and in 20 patients ( $26 \%)$, the protocol therapy was discontinued early (at 0.6 to 54.3 weeks) because of a worsening clinical course or death due to aspergillosis (itraconazole failure). Twenty-three $(30 \%)$ patients withdrew for other reasons including possible toxicity $(7 \%)$ and death due to another cause but without resolution of aspergillosis (20\%). Itraconazole failure rates varied widely according to site of disease and underlying disease group: $14 \%$ for pulmonary and tracheobronchial disease, $50 \%$ for sinus disease, $63 \%$ for central nervous system disease, and $44 \%$ for other sites; $7 \%$ in solid organ transplant, $29 \%$ in allogeneic bone marrow transplant patients, and $14 \%$ in those with prolonged granulocytopenia (median 19 days), $44 \%$ in AIDS patients, and $32 \%$ in other host groups. The relapse rates among those who completed therapy and those who discontinued early for possible toxicity were $12 \%$ and $40 \%$, respectively; all were still immunosuppressed. CONCLUSION: Oral itraconazole is a useful alternative therapy for invasive aspergillosis with response rates apparently comparable to amphotericin B. Relapse in immunocompromised patients may be a problem. Controlled trials are necessary to fully assess the role of itraconazole in the treatment of invasive aspergillosis.

Tnvasive aspergillosis is the most common lifethreatening invasive mould infection worldwide. It is a relatively common complication of profound neutropenia, bone marrow transplantation (BMT), solid organ transplantation, and chronic granulomatous disease and occurs sporadically in other immunocompromised patients such as those taking corticosteroids, those with diabetes, alcoholic patients, and patients with acquired immunodeficiency syndrome (AIDS). ${ }^{1,2}$

Invasive aspergillosis is often nosocomially acquired, sometimes associated with faulty or contaminated ventilation systems in hospitals and/or hospital construction projects. ${ }^{3}$ 
The treatment of invasive aspergillosis has, to date, been exclusively with amphotericin $B$, on occasion in combination with flucytosine and/or rifampin.,14-7 Early empirical treatment with high doses of amphotericin B (1.0 to $1.5 \mathrm{mg} / \mathrm{kg} /$ day) of pulmonary aspergillosis during neutropenia has lowered mortality in this group of patients from $80 \%$ to $100 \%$ in the early $1970 \mathbf{s}^{4,5}$ to as low as $13 \%$ in the late $1980 \mathrm{~s},{ }^{6}$ providing neutropenia resolves. Response rates in other host groups and in certain sites of disease are less satisfactory; mortality in patients with cerebral aspergillosis ${ }^{7}$ or pulmonary aspergillosis following allogeneic BMT exceeds $94 \%{ }^{8}$. Overall, in patients who are able to receive at least 14 days of amphotericin B therapy, there is a $55 \%$ response rate in all host groups at all sites of discasc. ${ }^{7}$ In all patients regardless of duration of therapy, the overall response rate with amphotericin B is probably $30 \%$ to $35 \%{ }^{7}$

Itraconazole is a new triazole antifungal agent with marked activity against Aspergillus species as demonstrated in vitro, ${ }^{9}$ in animal models, ${ }^{7}$ and in early patient studies of invasive aspergillosis. ${ }^{10-12}$ Based on encouraging preliminary data, the Mycoses Study Group studied the efficacy of itraconazole for invasive aspergillosis. We report here the first multicenter trial of the treatment of invasive aspergillosis.

\section{PATIENTS AND METHODS}

Patients were enrolled if they had evidence of definite or probable aspergillosis as per the definitions given below. Exclusion criteria included pregnancy and lactation, life expectancy judged to be less than 5 days, patients unable to take oral medication, and patients with only allergic bronchopulmonary aspergillosis, aspergillomas, or ocular aspergillosis disease. Patients with sinus aspergillosis who did not have histologic invasion of sinus mucosa or bone and patients with external otitis who did not have histologic evidence of tissue invasion were also excluded.

\section{Disease Definitions}

\section{No previous therapy}

1. Definite aspergillosis was defined as tissue histopathology showing septate, acute branching hyphae with or without a positive culture for Aspergillus species from the same site or, in the absence of histopathology, a positive culture from tissue obtained by an invasive procedure such as transbronchial biopsy or percutaneous needle aspiration.

2. Probable aspergillosis applied only to patients with pulmonary disease and was defined as patients with the chest radiographic appearance of new nodules or new cavities in the context of neutropenia, receipt of a cytotoxic agent for malignant or immunologic disease, corticosteroid dosage of more than 10 mg of prednisone or equivalent daily, or congenital or acquired immunodeficiency. In addition, all patients had to have two sputum cultures or one bronchoalveolar lavage (BAL), washings or brushings culture for Aspergillus species, or cytologic examination on BAL showing characteristic septate hyphae. Patients who were enrolled as probable aspergillosis were upgraded to definite aspergillosis if at autopsy or later surgical procedure, invasive aspergillosis was demonstrated histopathologically.

\section{Previous therapy failed}

Unless patients had experienced a failure of other therapy, the maximum alternative therapy for aspergillosis allowed prior to entry was (representing approximately 10 days of therapy at usual doses) less than or equal to $4.3 \mathrm{mg} / \mathrm{kg}$ of amphotericin B, less than or equal to $1.5 \mathrm{~g} / \mathrm{kg}$ of flucytosine, less than or equal to $30 \mathrm{~g}$ of miconazole, or less than or equal to $4 \mathrm{~g}$ of ketoconazole. Patients whose condition was deteriorating on therapy were enrolled if Aspergillus species were cultured from a biopsy specimen or percutaneous aspirate sample in the week prior to enrollment and there was prior histologic evidence of invasive aspergillosis (defined as definite aspergillosis). Those previously categorized as definite aspergillosis were enrolled if they had radiologic deterioration after a trial of therapy that was defined as a minimum of 3 weeks of at least $150 \mathrm{mg}$ of amphotericin B per week (redefined as probable aspergillosis).

The patients were classified according to the most prominent site of involvement. The majority of patients had only one site of aspergillosis, but there were some patients with both pulmonary and cerebral disease who were classified as having cerebral aspergillosis for the purposes of analysis.

\section{Treatment Protocol}

Itraconazole was administered orally as $100 \mathrm{mg}$ capsules with food. During the first 4 days of therapy, itraconazole $200 \mathrm{mg}$ was administered 3 times a day (loading dose); thereafter, the dose was $200 \mathrm{mg}$ twice daily. In patients not initially responding to therapy, a dose escalation to $200 \mathrm{mg} 3$ times a day was allowed if the serum concentration of itraconazole was less than $4 \mu \mathrm{g} / \mathrm{mL}$. This concentration was selected in an attempt to achicve in the serum a concentration that would exceed the minimum inhibitory concentration (MIC) of itraconazole for most aspergillus isolates in previous studies. ${ }^{9}$ No concurrent systemic antifungal therapy was allowed during itraconazole therapy.

Therapy was continued for a variable length of time depending on clinical response. In patients with a response to therapy, the minimum duration of therapy was 4 months and in many cases exceeded a year. Each investigator determined the duration of therapy according to clinical response and the need for continuing immunosuppression. 


\section{Assessment of Efficacy}

The following data were collected at baseline and at 2, 4, and 8 weeks and $3,6,9$, and 12 months thereafter while on therapy, at the end of therapy, and at three-monthly intervals after the completion of therapy for patients still alive. Symptoms and clinical signs were assessed at each evaluation point. Radiologic imaging was conducted at baseline and usually at each evaluation point. Computerized tomography and magnelic resonance scans were usually repeated at 2- to 3-month intervals. Invasive studies done at baseline for histopathology and culture were not usually repeated unless a surgical procedure was undertaken. Cultures from accessible sites such as sputum or urine were repeated frequently. Data on the underlying disease and immunocompromising factors including neutrophil counts, graft-versus-host disease (GVHD), doses of corticosteroid agents and other immunosuppressive agents, and episodes of rejection in solid organ transplant patients were collected at baseline and at each interval. Concurrent medication and possible side effects of itraconazole were also recorded at each evaluation point. Any surgical procedure undertaken as a therapeutic measure was recorded. Patient responses have been described overall regardless of surgery. All case record forms and in many cases radiographs were carefully reviewed together by two of the authors (DWD, JYL), and outcome assessments agreed together, often in consultation with the local investigator if necessary.

Responses at 12 weeks and end of treatment were categorized as follows: complete response: resolution of all attributable symptoms, signs, and radiographic and/or bronchoscopic abnormalities, if present at enrollment; partial response: major improvement (usually nearly complete) in attributable symptoms, signs, and radiographic and/or bronchoscopic abnormalities, if present at enrollment; stable disease: minor or no improvement in attributable symptoms, signs, and radiographic and/or bronchoscopic abnormalities but patient continued on therapy without deterioration; and failure: deterioration in attributable clinical and/or radiographic abnormalities necessitating alternative antifungal therapy or resulting in death.

Eight unevaluable patients did not meet the entry criteria.

Posttreatment follow-up data have not been included in response rates (in common with all prior literature on invasive aspergillosis). However, relapse was defined as the re-emergence of invasive aspergillosis after discontinuation of therapy following a complete, partial, or stable response, or following early withdrawal due to toxicity.

Death was categorized in two ways. Death was classified as being due to aspergillosis when an inexorable downhill course was noted with death pri- marily attributable to invasive aspergillosis. Death with aspergillosis was the assigned classification when patients died of another cause (clearly identifiable) but invasive aspergillosis was still present at the time of death as judged clinically, radiographically, or at autopsy. If the patient died of other causes and no aspergillosis was demonstrable at autopsy, the patient was classified by the last antemortem response, as described previously, and not as death.

Two anlalyses have been done with respect to failure. One uses the narrow definition of antifungal failure as given previously (itraconazole failure). The other is termed overall failure and is a broader definition that includes all patients with itraconazole failure as well as those with toxicity that resulted in the termination of therapy, death with but not due to aspergillosis, and inability to take oral medication and other adverse reasons for terminating the study.

\section{Statistical Analysis}

Response rates were estimated as the proportion of patients who achieved a complete or partial response. The $95 \%$ confidence intervals for the response rates were estimated using the normal approximation.

The chi-square test was used to compare response rates between patients who had received less than or equal to $4.3 \mathrm{mg} / \mathrm{kg}$ of amphotericin B and those who had not. Student's $t$-test was used to compare responders and nonresponders with respect to serum itraconazole concentrations.

\section{Laboratory Procedures}

Serum concentrations of itraconazole were assayed approximately 7 days after initiating therapy (assumed steady state concentration after loading doses) by bioassay at the Santa Clara Valley Medical Center as previously described. ${ }^{11}$ Trough concentrations were used for analysis if available. If multiple results were available, the mean trough concentrations are given for the first 2- to 3-month period of therapy. Susceptibility to itraconazole was determined by macrodilution broth tests at the Santa Clara Valley Medical Center as previously described.9 Resistance was defined as an MIC greater than 12.5 $\mu \mathrm{g} / \mathrm{mL}$, selected becausc this concentration cxcceds the mean serum concentration in previous studies. ${ }^{7}$

\section{RESULTS}

\section{Patient Population}

The characteristics of the 76 evaluable patients are shown in Table I. There was a slightly higher proportion of male patients with pulmonary aspergillosis but an equal number of male and female patients with extrapulmonary disease. There was a wide range of underlying conditions including AIDS, BMT, neutropenia, solid organ transplantation, diabetes melli- 


\begin{tabular}{|c|c|c|c|}
\hline \multicolumn{4}{|c|}{ Patient Characteristics } \\
\hline & $\begin{array}{c}\text { Pulmonary } \\
(\%)\end{array}$ & $\begin{array}{c}\text { Extra- } \\
\text { pulmonary } \\
(\%)\end{array}$ & $\begin{array}{l}\text { Total } \\
(\%)\end{array}$ \\
\hline $\begin{array}{c}\text { Total number } \\
\text { of patients }\end{array}$ & $51(67)$ & $25(33)$ & $76(100)$ \\
\hline Mean age $(y)$ & 47.5 & 48.9 & 48.0 \\
\hline Male sex & $36(71)$ & $13(52)$ & $49(64)$ \\
\hline \multicolumn{4}{|l|}{ Underlying diseases } \\
\hline Granulocytopenia* & $9(18)$ & $4(16)$ & $13(17)$ \\
\hline Bone marrow transplant ${ }^{\dagger}$ & $+6(11)$ & $2(8)$ & 8 (11) \\
\hline Solid organ transplant ${ }^{\ddagger}$ & $12(24)$ & $2(8)$ & $14(18)$ \\
\hline Corticosteroid therapy & $3(6)$ & $2(8)$ & $5(7)$ \\
\hline AIDS & $11(22)$ & $5(20)$ & $16(21)$ \\
\hline Other diseases" & $6(11)$ & $8(32)$ & $14(18)$ \\
\hline & $6(8)$ \\
\hline Totals & 51 & 25 & 76 \\
\hline \multicolumn{4}{|l|}{$\begin{array}{l}\text { Diagnosis of } \\
\text { aspergillosis }\end{array}$} \\
\hline Definite & $39(76)$ & $25(100)$ & $64(84)$ \\
\hline Probable & $12(24)$ & 0 & $12(16)$ \\
\hline \multicolumn{4}{|l|}{ Prior therapy } \\
\hline None & $22(43)$ & $8(32)$ & $30(39)$ \\
\hline $\begin{array}{l}\leq 4.3 \mathrm{mg} / \mathrm{kg} \\
\text { amphotericin } \mathrm{B}^{\S}\end{array}$ & $21(41)$ & $13(52)$ & $34(45)$ \\
\hline AMB failure/relapse & $8(16)$ & $4(16)$ & $12(16)$ \\
\hline \multicolumn{4}{|c|}{$\begin{array}{l}\text { Total includes } 5 \text { patients with acute myeloid leukemia, } 1 \text { with chronic lym- } \\
\text { phocytic leukemia and lymphoma, } 1 \text { with acute lymphoblastic leukemia, } 3 \\
\text { with lymphoma ( } 1 \text { ADS associated), and } 1 \text { patient each with pancytopenia, } \\
\text { myelodysplastic syndrome and aplastic anemia. } \\
\text { †Total includes } 7 \text { patients with allogeneic BMT and } 1 \text { with autologous BMT. } \\
\text { 'Total includes } 9 \text { patients with heart, } 2 \text { with heartlung, and } 1 \text { each with } \\
\text { single lung, kidney and liver transplants. } \\
\text { 'Total includes } 5 \text { patients with diabetes mellitus, } 2 \text { with chronic renal fail- } \\
\text { ure, and I each with systemic lupus erythematosus, low dose corticos- } \\
\text { teroid therapy, non-A non-B hepatitis and cirrhosis, prior spontaneous } \\
\text { pneumothorax, severe underlying right sided pulmonary fibrosis, radiation } \\
\text { damagc to lung postmastcctomy, and Shcehan's syndrome with hydrocor- } \\
\text { tisone replacement. } \\
\text { §Total includes } 1 \text { patient who also failed SCH } 39304 \text { and flucytosine. } \\
\text { AMB = Arripholericir B. }\end{array}$} \\
\hline
\end{tabular}

tus, and corticosteroid therapy. The majority of the patients (84\%) had definite aspergillosis. Twelve of 51 patients (24\%) enrolled with pulmonary disease had probable aspergillosis.

\section{Aspergillus Isolates and In Vitro Susceptibility}

In $57(75 \%)$ of the patients, an isolate of $A s-$ pergillus species was obtained. These were $A s$ pergillus fumigatus in 40 patients, Aspergillus flavus in 6, Aspergillus terreus in 3, and Aspergillus niger in 1, and in 7 patients, the isolate was not identified to species level. In 1 patient, 2 species were found. Forty isolates were available for susceptibility testing. Itraconazole MICs ranged from 0.4 to 3.1 $\mu \mathrm{g} / \mathrm{mL}$, with a median of $1.6 \mu \mathrm{g} / \mathrm{mL}$. The minimum fungicidal concentrations (MFC) ranged from 0.8 to $12.5 \mu \mathrm{g} / \mathrm{mL}$, with a median of $3.1 \mu \mathrm{g} / \mathrm{mL}$. Thus, no isolate was identified that was resistant to itraconazole. There was no difference in outcome related to the MIC or MFC value.

\section{Response Rates at 12 Weeks}

The overall response rate at 12 weeks was $32 \%$ ( $95 \%$ CI $22 \%$ to $42 \%$ ) (Table II). This is intended to be the lowest, most conservative estimate of successful therapy. Patients with pulmonary aspergillosis fared better than those with extrapulmonary disease. The subset of 13 patients with definite aspergillosis based on histology (no positive culture) fared less well (15\% response rate) than the overall group . There were no differences in the response between patients with definite and probable pulmonary aspergillosis (43\% and 45\%, respectively). Among the patients with extrapulmonary disease, there were no complete responders at 12 weeks and a considerably higher percentage of itraconazole failures than among the pulmonary group (36\% versus $10 \%$ ). The highest itraconazole failure rate was in those with aspergillosis of the central nervous system (CNS) (50\%).

Response rates according to underlying disease varied considerably at 12 weeks. The best results were seen in the group with solid organ transplants in which there was a $7 \%$ itraconazole failure rate at 12 weeks. In AIDS patients, the itraconazole failure rate was $25 \%$. In the granulocytopenia/cancer/BMT group, 19\% were classified as itraconazole failures. However, in this group, the failure rates were higher in the seven allogeneic BMT patients (29\%). In the seven patients with prolonged granulocytopenia (greater than or equal to 7 days, median 19 days), the itraconazole failure rate was $14 \%$. One patient, for example, had had an allogeneic BMT, remained neutropenic for 163 days, and was a complete responder, now continuing therapy. Among those patients with other underlying diseases, $20 \%$ had itraconazole failures and $28 \%$ had overall failures.

\section{End of Treatment Response Rates}

The overall response rate at the end of treatment was $39 \%$ (95\% CI $27 \%$ to $49 \%$ ). This figure includes the eight patients still on therapy. There are substantial differences in outcome depending on the precise time used for evaluation (Table II and Table III). Twelve weeks was initially selected as being a time that would be clinically meaningful. This proved to be the case for the solid organ transplant, BMT, neutropenic, and cancer patients since all itraconazole failures in these 35 patients occurred before 12 weeks, indeed all before 6 weeks. However, in the AIDS patients and those on corticosteroids or diabetic or without immunocompromising factors, many itraconazole failures occurred after 12 weeks.

Among the AIDS patients, the itraconazole failure rate was $25 \%$ at 12 weeks but $44 \%$ by the end of treatment, with the final patient experiencing a failure at 54 weeks. In those patients with no or mild immuno- 


\section{TABLE ॥}

Response and Failure at 12 Weeks (\%)

\begin{tabular}{|c|c|c|c|c|c|c|c|}
\hline & $\begin{array}{l}\text { Complete } \\
\text { Response }\end{array}$ & $\begin{array}{c}\text { Partial } \\
\text { Response }\end{array}$ & Stable & $\begin{array}{c}\text { Itraconazole } \\
\text { Failure }^{\dagger}\end{array}$ & $\begin{array}{c}\text { Failure for } \\
\text { Other Reasons }\end{array}$ & $\begin{array}{l}\text { Overall } \\
\text { Failure }\end{array}$ & Total \\
\hline \multicolumn{8}{|l|}{ Pulmonary aspergillosis* } \\
\hline All patients & $5(11)$ & $1 /(33)$ & $17(33)$ & $5(10)$ & $7(14)$ & $12(24)$ & 51 \\
\hline Granulocytopenia/cancer & $1(11)$ & $4(44)$ & $2(22)$ & $1(11)$ & $1(11)$ & $2(22)$ & 9 \\
\hline Bone marrow transplant & $1(17)$ & $2(33)$ & $1(17)$ & 0 & $2(33)$ & $2(33)$ & 6 \\
\hline Solid organ transplant & $2(17)$ & $5(42)$ & $3(25)$ & 0 & $2(17)$ & $2(17)$ & 12 \\
\hline AIDS & 0 & $2(18)$ & $5(45)$ & $2(18)$ & $2(18)$ & $4(36)$ & 11 \\
\hline Other & $1(8)$ & $4(31)$ & $6(46)$ & $2(15)$ & 0 & $2(15)$ & 13 \\
\hline \multicolumn{8}{|c|}{ Extrapulmonary aspergillosis } \\
\hline All patients & 0 & $3(12)$ & $9(36)$ & $9(36)$ & $4(16)$ & $13(52)$ & 25 \\
\hline CNS aspergillosis & 0 & $1(13)$ & $2(25)$ & $4(50)$ & $1(13)$ & $5(63)$ & 8 \\
\hline Sinus aspergillosis & 0 & $1(13)$ & $4(50)$ & $2(25)$ & $1(13)$ & $3(38)$ & 8 \\
\hline Other sites & 0 & $1(11)$ & $3(33)$ & $4(44)$ & $2(22)$ & $5(56)$ & 9 \\
\hline \multicolumn{8}{|l|}{ Host groups, all sites } \\
\hline All patients & $5(7)$ & $20(26)$ & $26(34)$ & $14(18)$ & $11(14)$ & $25(32)$ & 76 \\
\hline Granulocytopenia/cancer & $1(8)$ & $5(38)$ & $3(23)$ & $2(15)$ & $2(15)$ & $4(31)$ & 13 \\
\hline Bone marrow transplant & $1(13)$ & $2(25)$ & $1(13)$ & $2(25)$ & $2(25)$ & $4(50)$ & 8 \\
\hline Solid organ transplant & $2(14)$ & $5(36)$ & $4(29)$ & $1(7)$ & $2(14)$ & $3(21)$ & 14 \\
\hline AIDS & 0 & $2(13)$ & $7(44)$ & $4(25)$ & $3(38)$ & $7(44)$ & 16 \\
\hline Steroid therapy & 0 & $1(20)$ & $2(40)$ & $1(20)$ & $1(20)$ & $2(40)$ & 5 \\
\hline Mildly immunocompromised & ed 0 & $3(21)$ & $8(58)$ & $2(14)$ & $1(7)$ & $3(21)$ & 14 \\
\hline Not immunocompromised & $1(17)$ & $2(33)$ & $1(17)$ & $2(33)$ & 0 & $2(33)$ & 6 \\
\hline
\end{tabular}

TABLE III

End of Treatment Responses (\%)

\begin{tabular}{|c|c|c|c|c|c|c|c|}
\hline & $\begin{array}{l}\text { Complete } \\
\text { Response }\end{array}$ & $\begin{array}{c}\text { Partial } \\
\text { Response }\end{array}$ & Stable & $\begin{array}{c}\text { Itraconazole } \\
\text { Failure }^{\dagger}\end{array}$ & $\begin{array}{c}\text { Failure for } \\
\text { Other Reasons } \\
\end{array}$ & $\begin{array}{l}\text { Overail } \\
\text { Failure } \\
\end{array}$ & Total \\
\hline \multicolumn{8}{|l|}{ Pulmonary aspergillosis* } \\
\hline All patients & $17(33)$ & $8(16)$ & $3(6)$ & $7(14)$ & $16(31)$ & $23(45)$ & 51 \\
\hline Granulocytopenia/cancer & $5(56)$ & $1(11)$ & 0 & $1(11)$ & $2(22)$ & $3(33)$ & 9 \\
\hline Bone marrow transplant & $1(17)$ & $2(33)$ & $1(17)$ & 0 & $2(17)$ & $2(17)$ & 6 \\
\hline Solid organ transplant & $6(50)$ & $2(17)$ & $2(17)$ & 0 & $2(17)$ & $2(17)$ & 12 \\
\hline AIDS & 0 & 0 & 0 & $4(36)$ & $7(64)$ & $11(100)$ & 11 \\
\hline Other & $5(38)$ & $3(23)$ & 0 & $2(15)$ & $3(23)$ & $5(38)$ & 13 \\
\hline \multicolumn{8}{|c|}{ Extrapulmonary aspergillosis } \\
\hline All patients & $3(12)$ & $2(8)$ & $0(0)$ & $13(52)$ & $7(28)$ & $20(80)$ & 25 \\
\hline CNS aspergillosis & 0 & $1(13)$ & 0 & $5(63)$ & $2(25)$ & $7(8)$ & 8 \\
\hline Sinus aspergillosis & $2(25)$ & $1(13)$ & 0 & $4(50)$ & $1(13)$ & $5(63)$ & 8 \\
\hline Other sites & $1(11)$ & 0 & 0 & $4(44)$ & $4(44)$ & $8(89)$ & 9 \\
\hline \multicolumn{8}{|l|}{ Host groups, all sites } \\
\hline All patients & $20(26)$ & $10(13)$ & $3(4)$ & $20(26)$ & $23(30)$ & $43(56)$ & 76 \\
\hline Granulocytopenia/cancer & $6(46)$ & $2(15)$ & 0 & $2(15)$ & $3(23)$ & $5(38)$ & 13 \\
\hline Bone marrow transplant & $1(13)$ & $2(25)$ & $1(13)$ & $2(25)$ & $2(25)$ & $4(50)$ & 8 \\
\hline Solid organ transplant & $6(43)$ & $2(14)$ & $2(14)$ & $1(7)$ & $3(21)$ & $4(29)$ & 14 \\
\hline AIDS & 0 & 0 & 0 & $7(44)$ & $9(56)$ & $16(100)$ & 16 \\
\hline Steroid therapy & $3(60)$ & 0 & 0 & $1(20)$ & $1(20)$ & $2(40)$ & 5 \\
\hline Mildly immunocompromised & 2 (14) & $3(21)$ & 0 & $5(36)$ & $4(29)$ & $9(64)$ & 14 \\
\hline Not immunocompromised & d $2(33)$ & $1(17)$ & 0 & $2(33)$ & $1(17)$ & $3(50)$ & 6 \\
\hline
\end{tabular}

compromising underlying disease, the 12-week itraconazole failure rate rose from $20 \%$ to $35 \%$ at the end of study. Six patients with stable responses at 12 weeks subsequently had complete responses, and several other patients had partial responses and/or were continuing on therapy. Five patients with stable responses at 12 weeks subsequently experienced a failure of therapy.

With respect to organ involvement, itraconazole failure at the end of therapy was seen in $14 \%$ of pul- 


\begin{tabular}{|c|c|c|c|c|c|}
\hline \multirow[t]{2}{*}{ TABLE IV } & \multicolumn{4}{|c|}{ Reason for Discontinuation of Itraconazole at any Time } & \multirow[b]{2}{*}{$\begin{array}{c}\text { Total } \\
(\mathrm{N}=76)(\%\end{array}$} \\
\hline & $\begin{array}{l}\text { BMT/Cancer } \\
\text { Granulocytopenia } \\
(\mathbf{N}=\mathbf{2 1})\end{array}$ & $\begin{array}{l}\text { Solid Organ } \\
\text { Transplant } \\
(\mathbf{N}=14)\end{array}$ & $\begin{array}{c}\text { AIDS } \\
(\mathbf{N}=16)\end{array}$ & $\begin{array}{c}\text { Other } \\
\langle N=25\rangle\end{array}$ & \\
\hline Possible toxicity & 0 & $1^{*}$ & 2 & 2 & $5(7)$ \\
\hline Worsening clinical course & 1 & 1 & 5 & 5 & 12 (16) \\
\hline \multirow{2}{*}{\multicolumn{6}{|c|}{ Death due to other causes }} \\
\hline & & & & & \\
\hline a) With aspergillosis & 4 & 1 & 7 & 3 & $15(20)$ \\
\hline b) Without aspergillosis & 1 & 0 & 0 & 0 & $1(1)$ \\
\hline Inability to take oral medication & 1 & 0 & 0 & 0 & $1(1)$ \\
\hline Other & 0 & $1^{\dagger}$ & 0 & $1^{\ddagger}$ & $2(3)$ \\
\hline Total $(\%)$ & $10(48)$ & $4(29)$ & $16(100)$ & $14(56)$ & $44(58)$ \\
\hline \multicolumn{6}{|c|}{$\begin{array}{l}\text { Complete response and relapse (categorized as failure for other reasons because of possible toxicity). } \\
\text { † Candidemia on therapy. } \\
\text { † Noncompliance. }\end{array}$} \\
\hline
\end{tabular}

monary, $63 \%$ of CNS, $50 \%$ of sinus aspergillosis, and $44 \%$ of other disease sites.

The overall failure rate at the end of study was $56 \%$; $26 \%$ attributable to itraconazole failure and $30 \%$ to failure for other reasons. The rate of failure for other reasons was $31 \%$ in the pulmonary and $28 \%$ in the extrapulmonary groups. However, this figure varied from $14 \%$ in patients undergoing solid organ transplant to $56 \%$ in the AIDS patients, perhaps reflecting the severity of underlying disease. If all patients discontinuing study in the first 2 weeks are excluded, the itraconazole failure rate decreases to $22 \%$ and the overall failure rate to $51 \%$.

There were multiple reasons for discontinuing itraconazole early as shown in Table IV. Although the frequency of possible side effects was considerable (Table V), only five (7\%) patients discontinued therapy because of possible toxicity. Twelve patients (16\%) discontinued therapy with a worsening clinical course, and $8(11 \%)$ died of aspergillosis on itraconazole. Sixteen other patients died on therapy, 15 with aspergillosis and 1 without. Autopsies were not undertaken in all patients. Only one patient could not lake oral inedicadion. One solid organ transplant recipient with stable disease developed candidemia at 7 weeks and was switched to amphotericin B therapy; serum itraconazole concentrations were not obtained.

The duration of therapy was detcrmined for each patient by each local investigator, although 6 to 12 months was recommended on the basis of prior experience. In fact, among the 17 responders who have completed therapy as per protocol, the duration of therapy varied from 15 to 97 weeks (mean 46 ).

\section{Effect of Surgery on Outcome}

Among the eight patients with sinus aspergillosis, seven underwent surgery and one granulocytopenic patient did not. Of the seven who underwent surgery, two were cured and one had a partial response, three had failures of therapy, and one was unevaluable due to toxicity (peripheral edema) at 3 weeks. One pa- tient with pulmonary aspergillosis treated with steroids was cured with surgical resection of the lesion. One nonimmunocompromised patient with pulmonary aspergillosis had a repair of a bronchopulmonary fistula 2 weeks after enrollment, which did not alter the outcome; he had a failure of therapy. Another patient with peritoneal aspergillosis had a partial response with itraconazole and catheter removal and finally responded to amphotericin B with no relapse after the end of therapy. In no other patients was substantive surgery for aspergillosis undertaken during itraconazole therapy.

\section{Effect of Prior Therapy on Outcome}

Thirty-nine percent of the patients had received no prior therapy, and a further $45 \%$ had received less than or equal to $4.3 \mathrm{mg} / \mathrm{kg}$ of amphotericin B (Table I). There was no significant difference in outcome between these two groups by chi-square analysis $(P=$ 0.8 ). In addition, there were nine other patients who had a failure of amphotericin B therapy and one who had a failure with the experimental compound SCH 39304 and flucytosine. Five of these patients responded: two had stable disease, and three had a failure with itraconazole. Three other patients had a relapse after the end of amphotericin B therapy, and all three responded to itraconazole.

\section{Itraconazole Serum Concentrations and Response}

Serum concentrations of itraconazole were measured in 52 patients. Itraconazole trough concentrations were very variable as can be seen in the Figure. Higher mean concentrations were found in those patients who had a response by 3 months compared with those with stable disease and those with failures, but the differences were not statistically different $(P$ $=0.32$ ). Dose elevation from 450 to $800 \mathrm{mg} / \mathrm{d}$ was made in 11 patients usually at 4 to 8 weeks of study with increased serum concentrations documented in at least 2 patients. It is notable that there was a sub- 


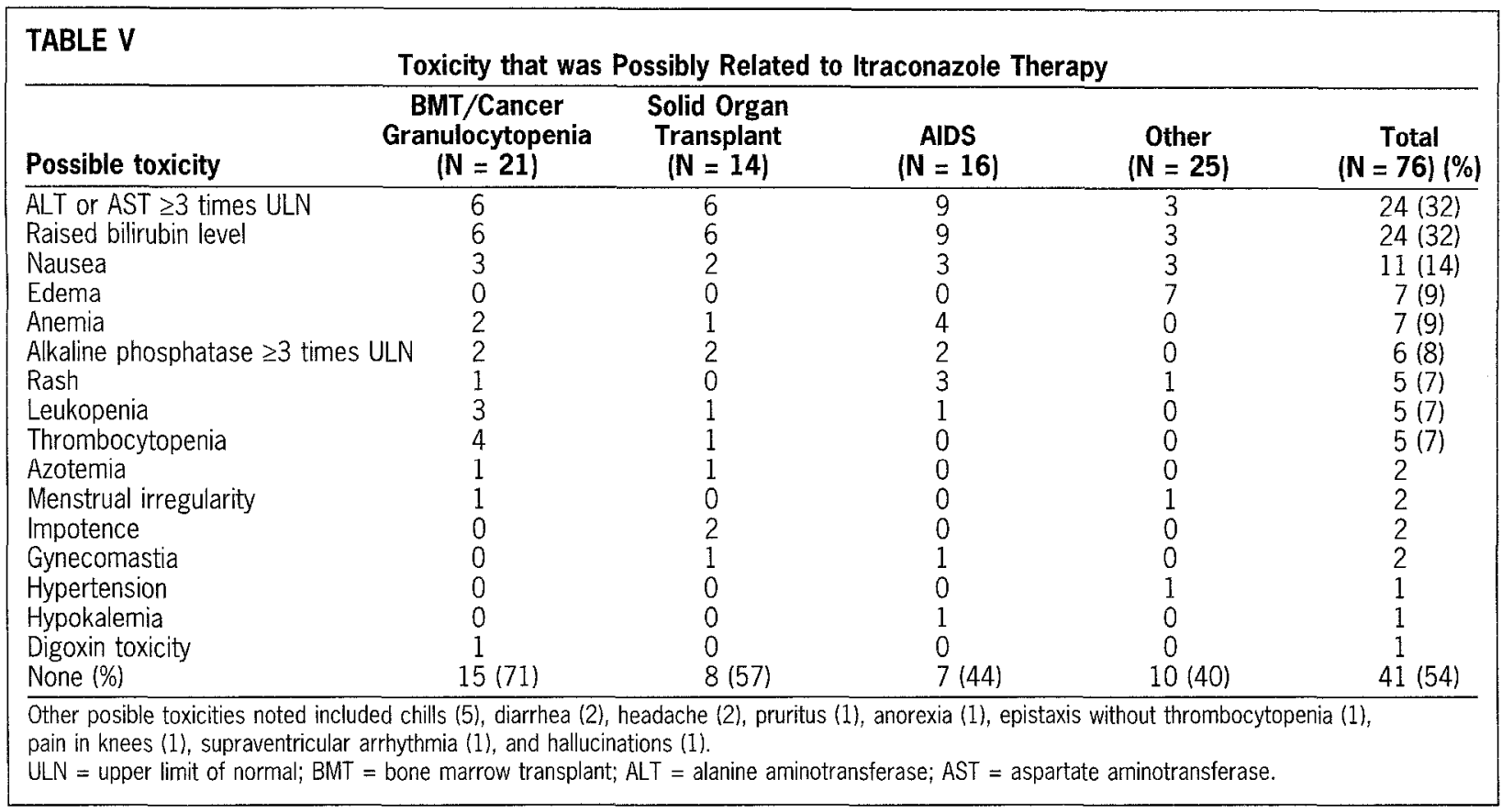

stantial group of patients who had a failure of therapy or a stable response with undetectable serum itraconazole concentrations. Drug interactions may have been responsible for low concentrations in some patients. No complete or partial responders had undetectable serum concentrations of itraconazole. It is also of interest that there were three failures with serum concentrations of 10 to $15 \mu \mathrm{g} / \mathrm{mL}$, well above the mean of patient samples in large studies ${ }^{7}$; one of these patients had CNS infection and two of the patients had AIDS.

\section{Posttreatment Follow-Up}

Of those who completed the treatment course, 3 of 25 (12\%) experienced a relapse. One had had a complete response, 1 a partial response, and 1 stable disease. Two of 5 other patients who had stopped itraconazole early for various reasons had a relapse (40\%). Of these 5 patients who had a relapse, all had continuing immunosuppression; 1 had pancytopenia, 1 AIDS, and the other 3 were solid organ transplant patients. Four had pulmonary aspergillosis and 1 tracheobronchial disease. Relapse occurred within 3 months of stopping itraconazole in all 5 patients. At least 3 other patients with partial responses at the end of therapy have not had a relapse more than 6 months after discontinuing itraconazole.

\section{Drug Interactions Noted}

Itraconazole may raise the serum concentrations of some drugs (eg, digoxin, cyclosporine, and terfenadine), and its concentration may be lowered to subtherapeutic concentrations by enzymes that induce the p450 enzymatic pathway (eg, rifampicin). There were a number of potentially detrimental drug inter- actions noted. One patient had an elevation in serum digoxin concentrations that may have contributed to a cardiac arrhythmia, which, along with numerous other problems, led to death at 3 weeks. The use of phenytoin for seizures in two allogenic BMT patients was probably detrimental in that these patients died at 5 and 7 days, respectively, after the initiation of therapy; in both patients, cerebral aspergillosis was documented at autopsy. Two patients who had a failure of therapy may have had an interaction with rifampicin: one with cutaneous aspergillosis, and the other with sinus aspergillosis. The patient with skin aspergillosis was granulocytopenic for 23 of 27 days before death. A heart transplant patient who had lumbar spine aspergillosis had low serum concentrations of itraconazole possibly attributable to concurrent ranitidine therapy and had a failure of therapy at 4 weeks. Eighteen palients were on cyclosporin therapy concurrently. Data about dose changes and serum cyclosporin concentrations were not regularly recorded. At least eight patients required a dose reduction of cyclosporin shortly after starting itraconazole.

\section{Adverse Events}

Toxicity possibly attributable to itraconazole is described in Table $V$. Although all possibly related side effects are shown, the conditions of the patients were in many cases so very complicated with multiple disease processes ongoing that it was difficult to attribute many of the adverse events directly to itraconazole. Thus, thrombocytopenia in the four BMT/ cancer/granulocytopenia patients was more likely related to cytotoxic chemotherapy/radiotherapy than to the drug. Likewise, the high incidence of liver function abnormalities in the AIDS patients was probably 


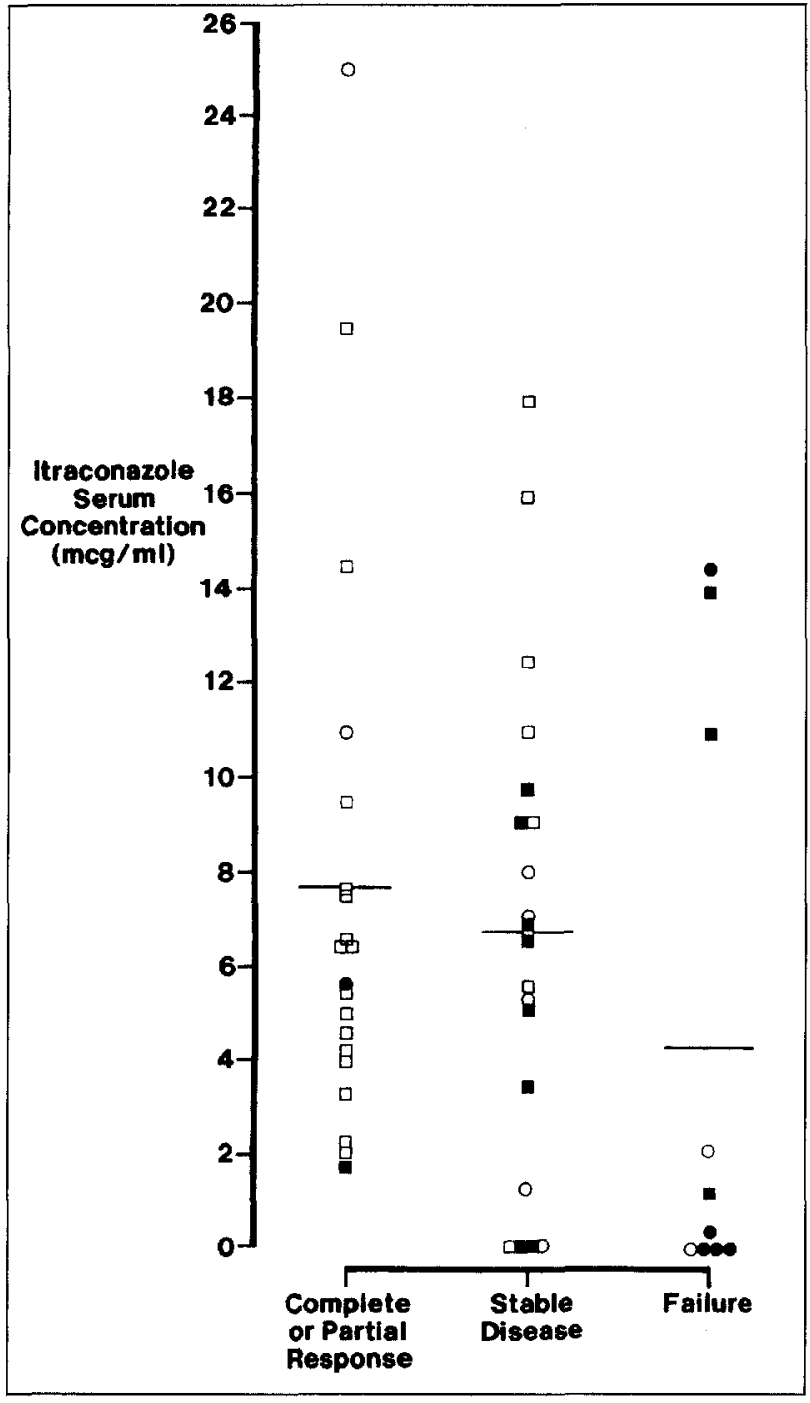

Figure. Serum itraconazole concentrations according to clinical response. $\square$ $=$ pulmonary aspergillosis in non-AIDS patients, $\mathbf{w}=$ pulmonary aspergillosis in AIDS patients, $\bullet=$ central nervous system aspergillosis, $\mathrm{O}=$ non-pulmonary non-CNS aspergillosis.

at least partly attributable to other disease processes. ${ }^{13}$ In addition, it is possible that some adverse events thought by the investigator not to be attributable to the drug were not noted in the case record form and so the record of adverse events is probably incompletc. It is, howcrer, of interest that edema and hypertension were only seen in the relatively nonimmunocompromised groups.

\section{COMMENTS}

There have been few large series of invasive aspergillosis published in which treatment outcome has been reported. One series of 25 renal transplant patients focused on the use of amphotericin B and surgery ${ }_{1}^{14}$ with favorable results. Recently published was a review of compassionate use itraconazole data worldwide that reported responses in about 300 patients with a variety of manifestations of aspergillosis treated with doses of itraconazole from 100 to 600 mg daily. ${ }^{15}$ We have prospectively studied treatment outcome in 76 patients with well-documented invasive aspergillosis, using loading doses of oral itraconazole followed by 400 to $600 \mathrm{mg}$ daily. Response rates at the end of treatment were best in those patients with pulmonary and tracheobronchial disease (49\%) and in granulocytopenic/cancer patients (61\%) and solid organ transplant patients (57\%). There was a high attrition rate due to other diseases or possible toxicity. Thus, itraconazole failure rates in these three groups at the end of study were $14 \%, 15 \%$, and $7 \%$, respectively. At the end of treatment, the response rate in the subset of definitc aspergillosis paticnts whose diagnosis was based on histology was slightly lower (23\%) than the overall response rate (39\%). It is not likely that many, if any, of the responding, histologically diagnosed patients had another fungal etiology confused with aspergillosis, since the likely candidates (Pseudallescheria boydii, Fusarium species, Penicillium species, etc.) are usually not responsive to itraconazole. Among the pulmonary patients, the responsive rate was not different for definite or probable disease, $48 \%$ and $55 \%$, respectively.

Failure rates with amphotericin B (with flucytosine in some cases) vary from $13 \%$ in neutropenic patients to around $94 \%$ in allogeneic BMT patients ${ }^{6}$ and those with cerebral aspergillosis. ${ }^{7}$ Previous data indicated that itraconazole (100 to $400 \mathrm{mg} / \mathrm{d}$ ) had a $19 \%$ failure rate in those who received at least 2 weeks of therapy, ${ }^{7}$ compared with $22 \%$ in this study. Many very ill patients including BMT and neutropenic patients were included in this study. The comparable failure rate of amphotericin B is $45 \%$, again excluding those who did not receive 2 weeks of therapy. ${ }^{7}$ However, these comparisons are at best rough as the present study excluded patients unlikely to be able to take oral therapy, and the previous literature review ${ }^{7}$ encompassed series and case reports with, probably, multiple different other biases. The case mix is also critical in arriving at these generalized figures given variable response rates in different host groups and body sites.

Categorization of response was occasionally problematic. Symptom resolution was helpful but of those with pulmonary aspergillosis only $44 \%$ manifested fever, $46 \%$ dyspnea, $48 \%$ cough, $33 \%$ pleuritic chest pain, and 20\% hemoptysis. The dramatic response and the relentless progression of disease leading to failure proved no challenge to classification. However, a large group of patients had intermediate responses, particularly at 12 weeks.

It is for this reason that we have introduced the categorization 'stable'. We believe that this response categorization is useful. In most untreated patients, invasive aspergillosis is a progressive infection with a 
time course varying according to host factors and therapy. Thus, in a nearly uniformly fatal disease if untreated, arrest of the infection with therapy is clinically useful, even if not ideal.

Very few previous case reports or series have reported long-term outcome. Both complete and partial responders were apparently cured, suggesting that persistent radiologic abnormality as seen in some partial responders may represent a radiologic scar rather than persistent aspergillosis. However, a relapse rate of $12 \%$ following months of therapy is of concern, and in patients with a truncated course of therapy, a $40 \%$ relapse rate is unacceptably high. All of the relapses occurred in patients with continuing immunosuppression, and, thus, prolonged therapy may be appropriate in this group. It is also possible that it is reinfection rather than relapse that has occurred, but we don't have paired isolates for typing ${ }^{16}$ in these cases.

There was no clear relationship between serum concentrations of itraconazole and outcome. Patients who had a failure of therapy were much more likely to have a low serum itraconazole concentration but there were three failure patients, two with AIDS and one with CNS disease who had apparently adequate concentrations. Eight patients apparently had undetectable serum concentrations, and all these patients either had a failure of therapy or stable disease. Thus, it would appear that a detectable serum concentration is essential for response. There is, however, no clear cutoff concentration that will always predict response, although generally higher serum concentrations appear to be desirable. The issue of the relationship between serum concentrations and outcome is further complicated because the bioassay employed in this study measures both itraconazole and its major active metabolite hydroxyitraconazole. ${ }^{17}$ It appears that, in different bioassay systems, hydroxyitraconazole produces a zone size considerably larger than that of itraconazole at the same concentration. Thus, detectable concentrations reported as itraconazole in this study represent the total measurable antifungal activity in serum.

Among the 40 aspergillus isolates from study patients we tested, none were resistant to itraconazole. Occasional resistant isolates have becn reported previously. ${ }^{11}$ Despite the lack of standardization of in vitro susceptibility testing of most antifungal agents, ${ }^{9}$ these data are somewhat reassuring at this time. Whether resistance will develop during therapy with long-term itraconazole use in invasive aspergillosis remains to be seen but no such cases have been reported to date. There is a definite need for continued vigilance in this area given the general propensity of micro-organisms to circumvent antimicrobial strategies.

Several patients failed therapy as a result of probable drug interactions with rifampicin and phenytoin.
The prior or concurrent use of these agents, in addition to carbamezepine and phenobarbital, ${ }^{18}$ should be a contraindication to the use of itraconazole for lifethreatening fungal disease. No interaction was noted in one patient treated with itraconazole in another study who was also receiving sodium valproate ${ }^{19}$; perhaps this is a preferable anticonvulsant agent to use with itraconazole. Uncertainty surrounds the concurrent use of $\mathrm{H}_{2}$ antagonists and itraconazole. Clinicians should be aware of a possible interaction leading to poor absorption of itraconazole and should monitor serum itraconazole concentrations more frequently and carefully in these patients. Likewise, solid organ transplant patients receiving cyclosporin who are to be given itraconazole should probably have an immediate cyclosporin dosage reduction followed by frequent monitoring. ${ }^{20}$

That there was no difference in outcome between the groups who had received no primary amphotericin B therapy and those who had received up to $4.3 \mathrm{mg} / \mathrm{kg}$ is also reassuring. There are theoretical considerations for possible antagonism of azoles and polyenes, but the sequential use of the agents as used in this study did not bear out this concern. This opens possibilities for therapeutic strategies based on sequential therapy, such as the initiation of amphotericin B therapy because of the necessity for intravenous medication followed by oral therapy with itraconazole. This study generated no data on true combination therapy, and, thus, we can make no comment on possible antagonism or synergy in this context.

This study corroborates results of smaller studies of the efficacy of itraconazole in invasive aspergillosis, particularly of the respiratory tract. ${ }^{10-12}$ However, failures do occur, and in many patients the disease is apparently arrested while on therapy without improvement. The lack of an intravenous formulation and the problematic drug interactions necessitate a continued search for additional antifungal agents with activity against Aspergillus species. Until the results of randomized trials are available, the best primary therapy for invasive aspergillosis remains a matter of conjecture. Itraconazole, however, represents a real step forward in that it is the first compound active after oral administration against this common opportunistic mycosis.

\section{REFERENCES}

1. Bodey GP, Vartivarian S. Aspergillosis. Eur J Cin Microbiol infect Dis. $1989 ; 8: 413-437$

2. Denning DW, Follansbee $S$, Scolaro $M$, et al. Pulmonary aspergillosis in AIDS. NEJM. 1991;324:654-662.

3. Walsh TJ, Dixon DM. Nosocomial aspergillosis: environmental microbiology, hospital epidemiology, diagnosis and treatment. Eur $J$ Epidemiol. 1989;5:131-142.

4. Young RC, Bennett JE, Vogel CL, et al. Aspergillosis. The spectrum of the disease in 98 patients. Medicine. 1970;49:147-173 
5. Aisner J, Schimpff SC, Wiernik PH. Treatment of invasive aspergillosis: relation of early diagnosis and treatment to response. Ann Intern Med. 1977;86:539-543.

6. Burch PA, Karp JE, Merz WG, et al. Favorable outcome of invasive aspergillosis in patients with acute leukemia. J Clin Oncol. 1987;5:1985-1993. 7. Denning DW, Stevens DA. Antifungal and surgical treatment of invasive aspergillosis: review of 2121 published cases. Rev infect Dis. 1990;12: 1147-1201.

8. Denning DW, Stepan DE, Blume KG, Stevens DA. Control of invasive pulmonary aspergillosis in a bone marrow transplant patient with oral itraconazole. J Infect. 1992;24:73-79.

9. Denning DW, Hanson LH, Perlman AM, Stevens DA. In vitro susceptibility and synergy studies of Aspergillus species to conventional and new agents. Diagn Microbiol infect Dis. 1992;15:21-34.

10. Viviani MA, Tortorano AM, Woestenborghs $R$, Cauwenbergh G. Experience with itraconazole in deep mycoses in northern Italy. Mykosen. 1987;30:233-244.

11. Denning DW, Tucker RM, Hanson LH, Stevens DA. Treatment of invasive aspergillosis with itraconazole. Am J Med. 1989;86:791-800.

12. Dupont B. Itraconazole therapy in aspergillosis: study in 49 patients. J Am Acad Dermatol. 1990;23:607-614.

13. Stevens DA, Greene SI, Lang OS. Thrush can be prevented in patients with acquired immunodeficiency syndrome and the acquired immunodeficiency syndrome-related complex. Arch Intern Med. 1991;151:2158-2464.
14. Weiland D, Ferguson RM, Peterson PK, et al. Aspergillosis in 25 renal transplant patients. Epidemiology, clinical presentation, diagnosis, and management. Ann Surg. 1983;198:622-629.

15. De Buele K, De Doncker P, Cauwenbergh G, et al. The treatment of aspergillosis and aspergilloma with itraconazole, clinical results of an open international study (1982-1987). Mycoses. 1988;31:476-485.

16. Denning DW, Clemons KV, Hanson LH, Stevens DA. Restriction endonuclease analysis of total cellular DNA of Aspergillus fumigatus isolates of geographically and epidemiolngically diverse origin. J Infect Dis. 1990;162:1151-1158

17. Hostetler JS, Heykants J, Clemons KV, et al. Bioassay-chromatography discrepancies explained by metabolism of itraconazole to hydroxyitraconazole: studies of interpatient variations in concentrations. Antimicrob Agents Chemother. 1993;37:2224-2227.

18. Tucker RM, Hanson LH, Denning DW, et al. The interaction of azoles with rifampin, phenytoin and carbamazepine: in vitro and clinical observations. Clin Infect Dis. 1992;14:165-174.

19. Denning DW, Tucker RM, Hostetler JS, et al. Oral itraconazole therapy of cryptococcal meningitis and cryptococcosis in patients with ADS. In: Vanden Bossche H, Mackenzie DWR, Cauwenbergh G, et al, eds. Mycoses in AIDS patients. New York: Plenum Press: 1990; 305-324.

20. Kramer MR, Marshall SE, Denning DW, et al. Drug interaction between cyclosporin and itraconazole in heart and heart-lung and lung transplant recipients with fungal disease. Ann Intern Med. 1990;113:327-329. 